\title{
Drying Kinetics of Papaya Slices in Microwave Intermittent Dryer
}

$$
\begin{aligned}
& \text { Jian-Wu DAl } \\
& \text {, a , Wen } \text { QIN }^{2, b} \text {, Zhi-Jun WU } \\
& \text { ZHANG } \\
& \text {, a a }
\end{aligned}
$$

${ }^{1}$ College of Mechanical and Electrical Engineering, Sichuan Agricultural University, 625014, Ya'An, Sichuan Province, China

${ }^{2}$ College of Food Science, Sichuan Agricultural University, 625014

\author{
Ya'An, Sichuan Province, China \\ adaijianwu@126.com, b420942276@qq.com
}

\begin{abstract}
Keywords: papaya slice, microwave intermittent drying, characteristic, drying quality
Abstract. This paper studies the effect of different microwave power levels, heating time, intermittent time and slice thickness on the drying kinetics of papaya slices. The influence towards the color appearance, the papaya microwave intermittent drying characteristics and the tendency of water loss were obtained. The results indicated that the drying rate of papaya during the microwave intermittent drying consistently declined with the change of microwave power levels. Meanwhile, the paper made comparison of the papaya drying qualities within different factors, which providing reference for the later practical using.
\end{abstract}

\section{Introduction}

Papaya is one of the well-known fruits in Ling'nan area located in the southern area of China, recognizing as the King of fruits. With sweet flesh, soft taste, unique fragrant aroma and high nutritional value, it can enhance human immunity and has antibacterial effect as well. Papaya was widely planted in China with the area about 11,000 hectares. Nowadays, the annual output of papaya is about 920,000 tons in China [1]. With the development of people's living level, the consumption of papaya has been a hot spot and become more and more popular in our country. The papaya cultivation efforts also increase continuously. Fresh papaya has a high moisture content approximately $80 \%$ (wet basis), which favors the growth of microorganisms. Furthermore, the high moisture content can easily enhance enzymatic and non-enzymatic reactions, resulting in rapid deterioration of fresh papaya quality and thus lead to a reduction in the possible medicinal and commercial value [2]. At present, choosing the best parameters of the drying technology to process fresh papaya would be of great importance and necessity.

Domestic dehydration products of papaya in the market have not been found frequently. Most manufacturers fabricated it into dried fruits and canned products, which still stayed on the more traditional drying methods. Huang Wei-ping et al. studied the characteristics of papaya by hot-air drying technology [3]. Gao He et al. investigated the papaya drying characteristics processed by hot-air drying and analyzed the effect of infrared radiation on the quality of dried papaya slices [4]. Waljszewskj $\mathrm{K}$ et al. studied on the osmotic dehydration of papaya by hot air drying. The results showed that sucrose concentration and PH value on the papaya color changes with no apparent effect, and the maturity of the fruits before processing is a very important factor [5].

As a new type of microwave drying technology, the microwave intermittent drying has some unique advantages such as high efficiency and rapid heating capacity. This new technology has been successfully applied into the ginkgo, grains and other agricultural products processing, for papaya dry application it has not yet been reported. Therefore, further study of microwave intermittent drying characteristics of papaya and gaining a better quality of the process parameters has a very important significance. 


\section{Materials and methods}

\section{Raw material}

Fresh papayas were purchased in shopping malls in Ya'an city and selected fully fresh test materials with matured product, uniform size, no insect pests and without mechanical damage. The samples were checked carefully to discard spoiled fruit in order to prevent contamination by bacteria or fungi.

\section{Experimental setup and procedure}
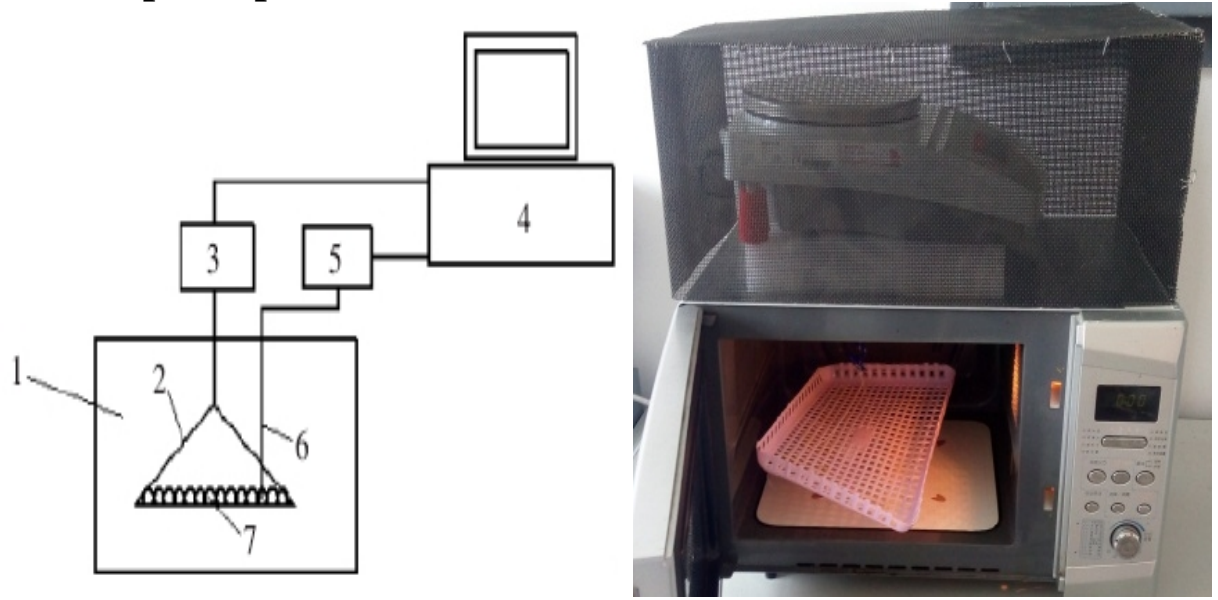

Fig. 1, The structure of microwave intermittent drying test device

Microwave intermittent drying test device as shown in fig. 1. Microwave intermittent dryer was an experimental platform built on the basis of microwave oven. Continuous emission of microwaves can make fresh agricultural products or fruit surfaces drying. So it was the primary task to carry out intermittent control of microwave experiments. The whole drying process to the weight of the material had a real-time grasp, which was the weight of the material or the dehydration of the material over time with a real-time response. Therefore, a hole was drilled on the basis of the microwave oven. Then the material tray was lifted by the balance. Due to the discharge reaction, the material tray and wire cannot be metal. Now, in this paper, cotton and plastic tray were used as the modified material. The tray was hung on the hook of the balance by cotton thread. And the weight of the balance was read in real time to reflect a change in the state of material dehydration. As a hole in the microwave oven, microwave leakage may occur. And this kind of discharge reaction can be avoided by wire screen. In order to play the role of shielding, a very tight wire net was stuck on the hole.

The preliminary experimental results showed that the power of batch microwave drying test from high to low was $700 \mathrm{~W}, 600 \mathrm{~W}, 450 \mathrm{~W}$ and $250 \mathrm{~W}$, respectively. Fresh-cut papaya slices were divided into four groups. A set of trials contained four levels, with four factors at four levels. The influence factors of papaya slices drying test were microwave power, heating time, slice thickness and interval time. The above four factors were used as variables to study the drying characteristics of papaya slices in different conditions and to analyze the specific influence of each factor and level. After each $30 \mathrm{~min}$, then weigh and recorded the test data during the experiment. And observe the quality changes of the sample in the experiment. When the papaya slices drying to moisture content was less than $15 \%$, the experiment was ended.

Table 1, Design for experiments with drying parameters included

\begin{tabular}{ccccc}
\hline \multirow{2}{*}{ Influence factor } & \multicolumn{4}{c}{ level } \\
\cline { 2 - 5 } & 1 & 2 & 3 & 4 \\
\hline microwave power /W & 250 & 450 & 600 & 700 \\
slice thickness /mm & 4 & 6 & 8 & 10 \\
heating time/s & 3 & 4 & 5 & 6 \\
interval time/s & 90 & 100 & 110 & 120 \\
\hline
\end{tabular}




\section{Calculation of moisture effective diffusivity}

The moisture ratio $(M R)$ of papaya samples during drying experiments was calculated using Eq. 1 .

$$
M R=\frac{M_{t}-M_{e}}{M_{o}-M_{e}}
$$

Where $M_{0}$ is the initial moisture content, $M_{e}$ is the equilibrium moisture content and $M_{t}$ is the moisture content at time t. The values of the equilibrium moisture content $M_{e}$ are relatively small compared to $M_{t}$ or $M_{0}$. Thus the Eq. 2 can be written in a more simplified form as follows [6-8]:

$$
D R=\frac{M_{t_{1}}-M_{t_{2}}}{t_{2}-t_{1}}
$$

The moisture effective diffusivity $\left(D_{e f f}\right)$ is an important transport property in describing drying characteristics of food and other materials, being a function of temperature and moisture content [9]. The Fick's second law of diffusion equation has been widely used to describe the drying process during the falling rate period [10-13]. Logarithmic simplification of Eq. 2 leads to a linear form Eq. 3:

$$
\ln (M R)=\ln \left(\frac{6}{\pi^{2}}\right)-\left(\pi^{2} \frac{D_{e f f} t}{r_{o}{ }^{2}}\right)
$$

By plotting the measured data plotted in a logarithmic scale, the effective moisture diffusivity was calculated as the slope of the line equal to the coefficient.

\section{Results and discussion}

Effect of different power level on drying rate of papaya slices

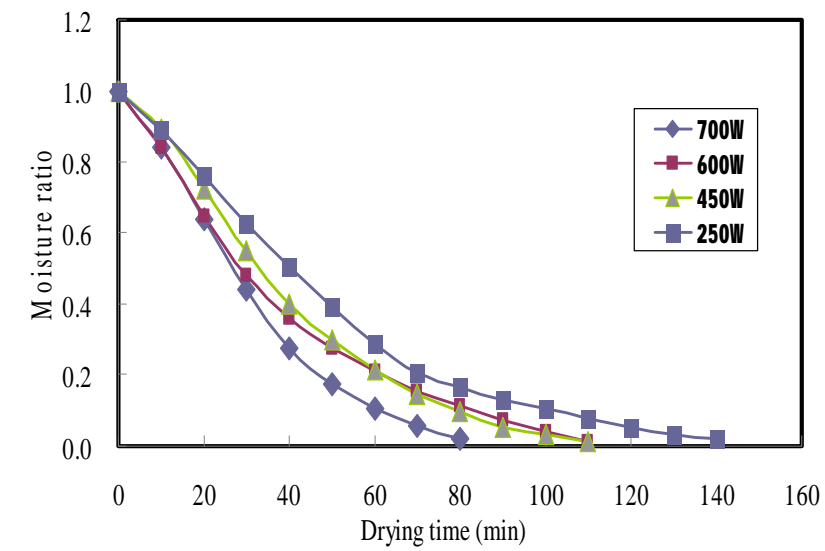

(a)

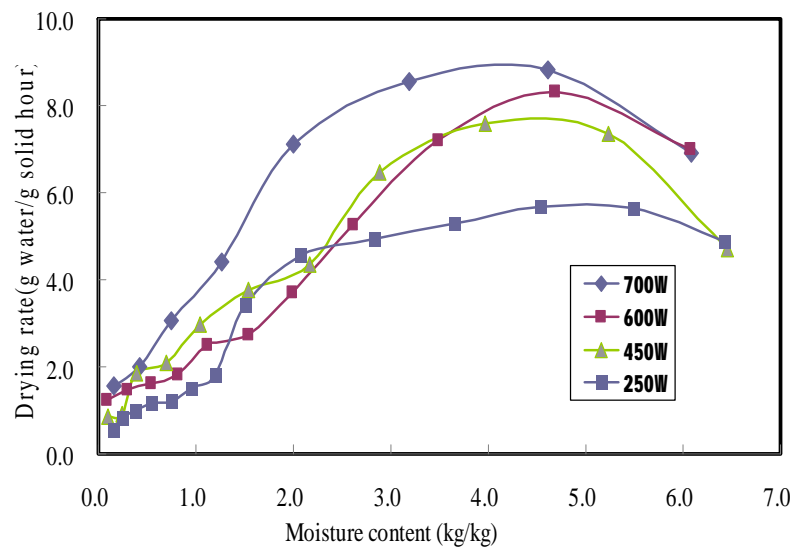

(b)

Fig. 2, Drying kinetics of papaya slices at different microwave power level with the heating/intermittent time of 4/100s and slices thickness of $6 \mathrm{~mm}$

The fig. 2 a showed that when the microwave power became stronger, the curve was much steep. It implied that the microwave strength increased as the moisture ratio decreased. In the drying stage, microwave drying curve became flatter and more difficult. With the increase of power level, more moisture molecules started to diffuse slowly, which positively correlation to the moisture ratio was dropping with the microwave power. But when the power was lower than $250 \mathrm{~W}$, the drying time increased greatly. The test results showed that the measured power was too low when the temperature rises very slowly. The reason may be that at the early stage of drying process, the papaya slices with high moisture content got large driving force and the high moisture removal rate. With the decrease of moisture content, the moisture molecule escaped from papaya became much more difficult from the surface of papaya slices, which leaded to the decrease of water removal rate.

It can be seen from fig. $2 \mathrm{~b}$, when the power level was at $700 \mathrm{~W}, 600 \mathrm{~W}$ and $450 \mathrm{~W}$ during this experiment, there was no constant speed stage during the whole drying process, only classified into the acceleration phase and the deceleration phase. The greater the power was, the greater the drying rate. The drying time changed from accelerating stage into the deceleration stage went down 
according as the microwave power decreases. When the power was less than $250 \mathrm{~W}$, the drying tendency of papaya slices changed, which was divided into preheating stage, constant speed stage and reduction stage processes. Along with the decrease of microwave power, the constant speed and deceleration process would significantly extend. The dehydration process showed quickly variation in the preheating stage, but once they reach the maximum water loss rate of papaya, material containing the water was too low to be guaranteed. At the same time, the constant speed stage during papaya drying process did not exist when the microwave power was at high level, while the microwave power had a significant effect on the drying rate.

Effect of different heating time on drying rate of papaya slices

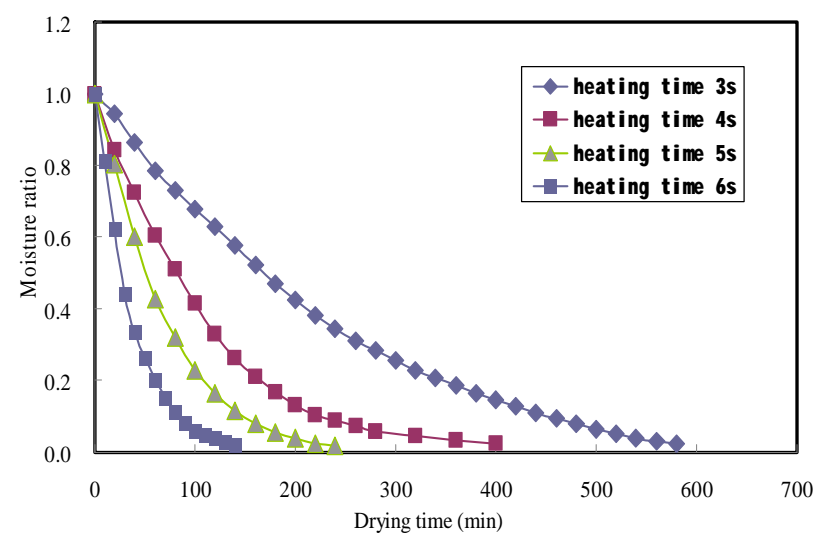

(a)

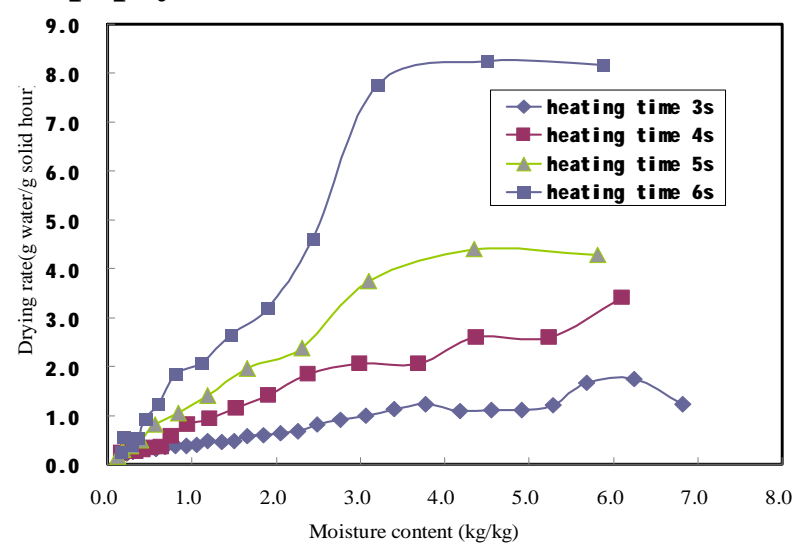

(b)

Fig. 3, Drying kinetics of papaya slices at different heating time with the intermittent time of $90 \mathrm{~s}$ at $700 \mathrm{~W}$ and thickness of $4 \mathrm{~mm}$

The fig. 3a showed that the drying curve became more steeps with the increase of heating time. And the required time drying to the safe water content would drop off as the water ratio decreased rapidly. The fig. $3 \mathrm{~b}$ showed that the papaya slices in this experiment stayed the same traditional tendency, generally included acceleration section, constant speed section and falling rate section. But the time of duration in the stage of accelerated section was very short, which prolong the heating time. The water loss rate accelerated with the increase of microwave power.

\section{Effect of different intermittent time on drying rate of papaya slices}

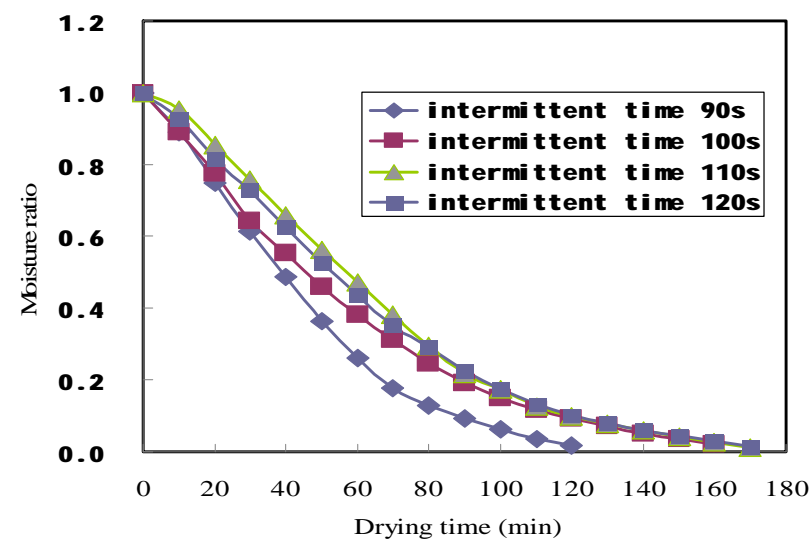

(a)

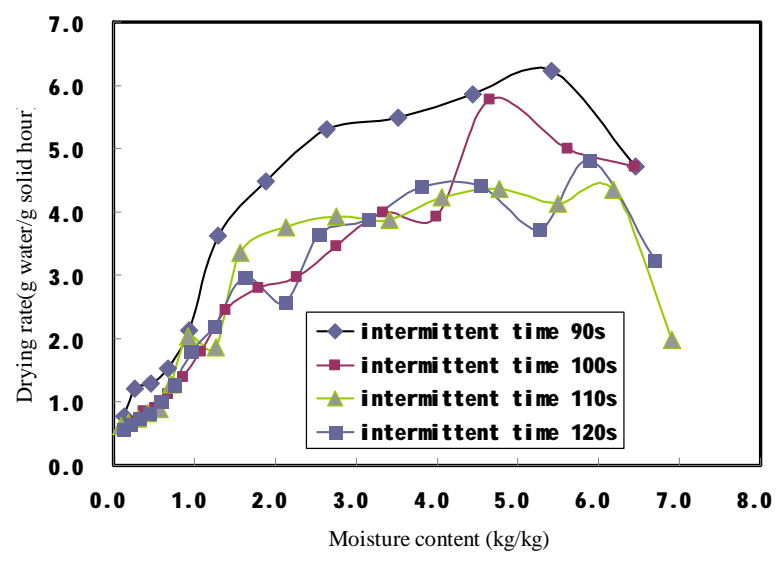

(b)

Fig. 4, Drying kinetics of papaya slices at different intermittent time with the heating time of $6 \mathrm{~s}$ at $700 \mathrm{~W}$ and thickness of $10 \mathrm{~mm}$

The fig. 4a showed that the whole drying time increased significantly with the extension of intermittent time, but the overall performance of drying quality had reached the requirements. The processing time would be extended along with the changes of the intermittent ratio. The longer the intermittent time, the moisture diffusion rate of declining speed would become much smaller. At the same time, the dehydration of papaya slices would get more difficulty. 
The fig. $4 \mathrm{~b}$ showed that the intermittent drying difference with general drying was in constant speed stage, the drying rate fluctuations. Experimental results showed that the setting of intermittent time parameter can effectively avoid local overheating phenomenon in the whole drying process of papaya slices.

Effect of different slice thickness on drying rate of papaya slices

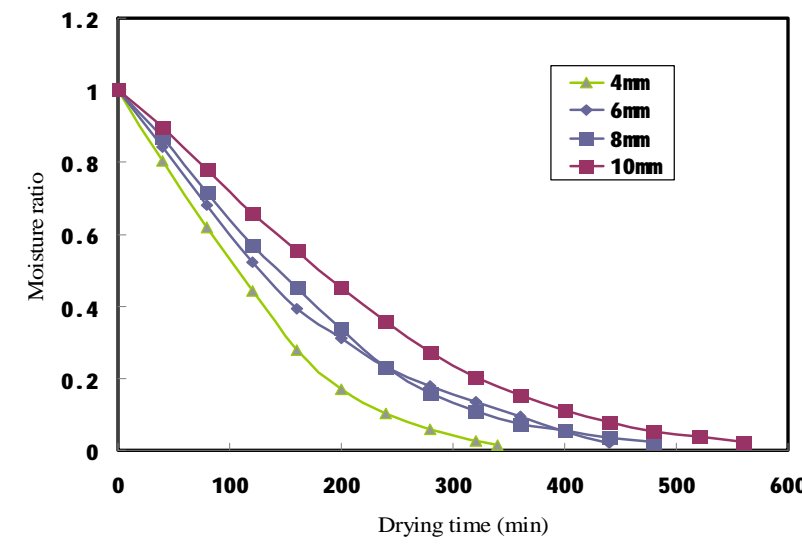

(a)

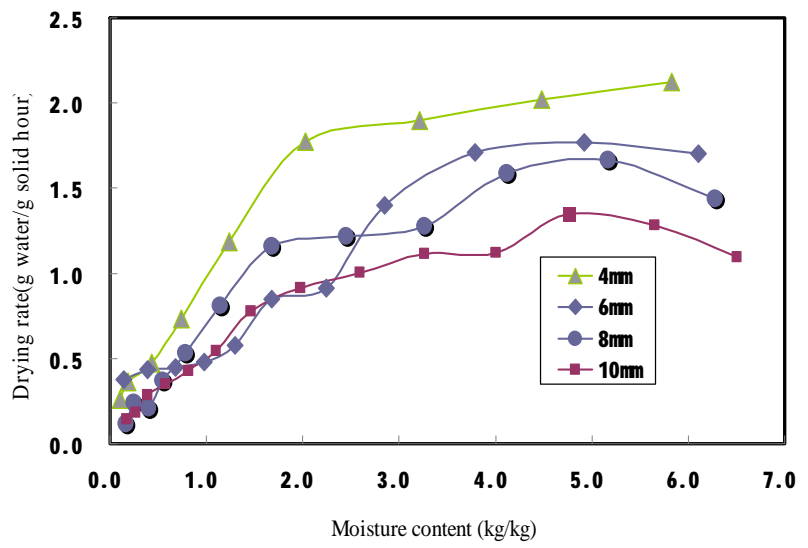

(b)

Fig. 5, Drying kinetics of papaya slices at different slice thickness with the heating/intermittent time of $6 / 110 \mathrm{~s}$ at $700 \mathrm{~W}$

The fig. 5a showed that the slice thickness had significantly effect on the change tendency of water ratio. With the increase of slice thickness, the internal moisture diffused to the surface during an extended process. In the end of the drying section, the falling speed of moisture ratio slowed down. This might be because along with the moisture content continuously declined, it became more difficult to lose the water from drying samples.

The fig. $5 b$ showed that between the different slice thicknesses under the same condition, the moisture falling rates were different from each other. When the slice thickness was larger, the papaya slices quality was much more easily affected. This mainly due to that the whole drying process with drying to the same moisture content needed more time. While the slice thickness was smaller, the microwave energy during the process of penetration degree would be reduced. And the center could accept more microwave energy, which leaded to heating overall more quickly.

\section{Conclusions}

(1) The drying characteristics of intermittent microwave drying of papaya could be divided into three sections, such as acceleration stage, constant speed stage and speed down drying stage. Microwave power, heating time, intermittent time and slice thickness with four levels were investigated on the drying process. Both of the above factors had negative effects on reduce of the whole drying process of papaya slices.

(2) The drying rate of papaya during the microwave intermittent drying consistently declined with the change of microwave power levels. When the value of power was at a low level, the duration time of constant rate drying period was significantly extended and the drying rate was small.

\section{Acknowledgements}

The authors gratefully acknowledge the Natural Science Project of Sichuan (No.035Z1986) for providing financial support to this investigation.

\section{References}

[1] Huang Weiping. Production of papaya[J]. Guangxi Agricultural Journal, 2011(2): 37-39. 
[2] E. K. Akamine, T. Goo. Relationship between surface color development and total soluble solids in papaya[J]. Honic. Science, 2011, 6(6): 567-569.

[3] J. E. Brekke, c. G Cavaketto, R. H. Nakayama Tbm Suehisa. Effect of storage temperature and container lining on some quality attributes of papaya nectar[J]. Journal of Agricultural Food Chemistry, 2013(21): 566-570.

[4] H T. Chan. Development of off-odors and of flavors in papaya puree[J]. Journal of Agricultural Food Chemistry, 2010(5): 355-360.

[5] Xu Yanyang. Ocular foreign research status of combined drying (on) papaya slices[N]. China food daily, 2003.07.24.

[6] Lu Chuanliang, Tong silver hung, Zhang Shi. A pearl polishing material with corn cob and the preparation method of papaya[P]. Chinese.CN101906271A.2010.

[7] Huang Xiaodong. Study on superfine pulverization technology of Rhizoma Coptidis[D]. East China University of Science and Technology, 2011.

[8] Ceng Qingxiao. Principle of food processing and preservation [M]. Beijing: Chemical Industry Press, 2002.

[9] Gunasekaran S. Pulsed microwave-vacuum drying of food materials[J]. Drying Technology, 2014, 17(3): 395-412.

[10] Krokida M K, Maroulis Z B. Effect of microwave drying on some quality properties of dehydrated products[J]. Drying Technology, 2008, 17(3): 449-466.

[11] Venkatachalapathy K., Raghavan G S V. MicmwaVe drying of whole sliced and pureed strawberries[J]. Journal of Agricultural Engineering, 9(1): 29-39.

[12] He Jia. Microwave drying vegetable and fruit quality of processed products[J]. Food Industry, 2002, 34(7): 47-57.

[13] Zhu Zhengliang, Fan Jian. Microwave drying of dry bracken research[J]. Journal of Yunnan Agricultural University, 2012, 17(3): 264-268. 